\title{
La participation publique dans la gestion des forêts du Québec : Réorganisation de la concertation régionale dans un contexte institutionnel en transition
}

\author{
par Jonathan Tardif, 1,"* Jean-François Bissonnette ${ }^{1}$ et Jérôme Dupras ${ }^{1}$
}

\begin{abstract}
RÉSUMÉ
La gouvernance forestière au Québec a subi de multiples mutations au cours des dernières décennies. D’un régime forestier qui favorisait les acteurs industriels, le gouvernement québécois a progressivement intégré toute une variété d’acteurs locaux et régionaux. Au printemps 2015, le gouvernement adoptait cependant un projet de loi entraînant labolition de plusieurs structures de développement régional, notamment les Conférences régionales des élus (CRÉ) qui étaient investies de responsabilités en ce domaine. Cet article présente les résultats d'une étude visant à mieux comprendre les impacts de cette décision sur la participation publique au sein des lieux de concertation propres au milieu forestier. La recherche s'appuie sur une analyse documentaire ainsi que sur 37 entretiens semi-directifs réalisés dans quatre régions du Québec. Parmi les constats qui émergent, mentionnons le désir quasi unanime de maintenir une concertation régionale autour des enjeux forestiers et la confiance limitée des intervenants quant à la volonté et la capacité des acteurs municipaux de reprendre le flambeau. Si de nombreux mécanismes demeurent en place pour assurer la participation des intervenants forestiers et de la société dans son ensemble, l’abolition des CRÉ restreint dans certaines régions les canaux de participation et de communication.
\end{abstract}

Mots clés: participation publique, gestion des forêts, décentralisation, lieux de concertation, Québec, CRRNT, Tables GIRT, loi 28

\section{ABSTRACT}

Forest stewardship in Québec has gone through several mutations during the last decades. From a forest regime that favoured the industrial stakeholders, the Government of Québec has progressively integrated a large scope of local and regional stakeholders. However in the spring of 2015, the Government of Québec adopted a legislation resulting in the abolition of several regional development structures, in particular the regional conferences of elected officials (CRE) which were invested with specific responsibilities in this matter. This article presents the results of a study carried on to better understand the impacts of this legislation on public implication in consultation forums dedicated to the forest. The research is based on a literature review and on 37 semi-structured interviews made in four regions of the province. The findings indicated a quasi unanimous desire to maintain regional consultation on forest issues and a limited stakeholders confidence regarding the willingness and the ability of municipal stakeholders to take up the torch. Even though numerous mechanisms are still in place to ensure forest stakeholders participation and the implication of the community in general, the CRE abolition is restricting in several regions the participation and communication channels.

Keywords: public participation, forest management, decentralisation, consultation forums, Québec, CRRNT, Tables GIRT, Bill 28

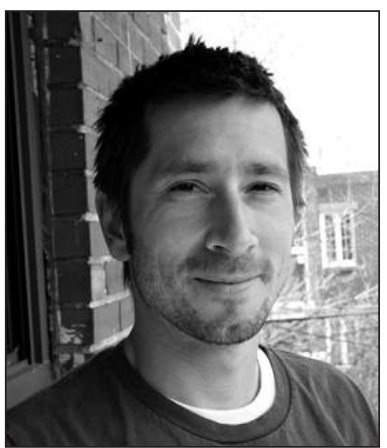

Jonathan Tardif

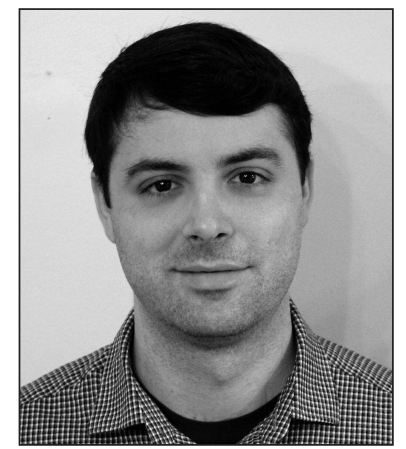

Jean-François Bissonnette

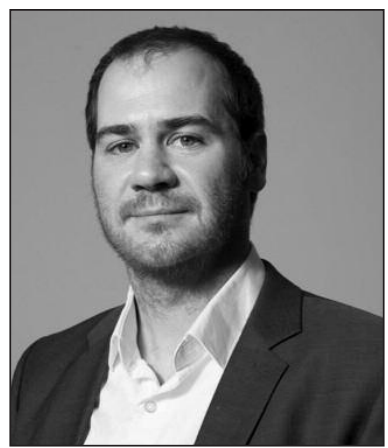

Jérôme Dupras

\footnotetext{
${ }^{1}$ Institut des Sciences de la Forêt Tempérée, Université du Québec en Outaouais; Email/Courriel: jonathan_tardif@yahoo.ca
} 


\section{Introduction}

La forêt joue un rôle clé dans le développement économique et social du Québec depuis le 19ème siècle et constitue un aspect incontournable de son organisation territoriale (Hardy et Séguin 2011). Cependant, la gouvernance forestière au Québec a subi de multiples mutations au cours des dernières décennies, suivant ainsi une tendance mondiale où on assiste à une plus grande décentralisation de la gestion et à une participation accrue du public dans ce secteur (McGurk et al. 2006, Agrawal et al. 2008, Blais et Boucher 2013).

Jusquau milieu des années 1980, les deux principaux acteurs impliqués dans la gestion des forêts québécoises étaient les grandes entreprises privées, responsables de la gestion des forêts, et l'État qui imposait alors peu de contraintes (Blais et Chiasson 2005). Cétait un modèle plutôt centralisé qui laissait peu de place aux autres utilisateurs de la forêt (Chiasson et al. 2013). Porté par des demandes sociales et des pressions économiques croissantes, le gouvernement québécois va revoir son régime forestier en profondeur à deux reprises (1986 et 2013) afin notamment de modifier le partage des rôles et des responsabilités entre les acteurs territoriaux. Ainsi, d'un régime forestier qui favorisait les grands acteurs industriels, le gouvernement québécois a peu à peu intégré toute une variété d'acteurs locaux et régionaux dans la prise de décision en matière de forêt (Chiasson et Leclerc 2013). Plusieurs instances de concertation ont été mises en place ces dernières années dans chacune des régions afin de favoriser le dialogue et la collaboration dans ce secteur, notamment les tables locales de gestion intégrée des ressources et du territoire (Tables GIRT), qui collaborent avec le gouvernement à la mise en place des plans d’aménagement forestier intégré $(\mathrm{PAFI})^{2}$, et les Commissions régionales sur les ressources naturelles et le territoire (CRRNT), où se discutent les grandes orientations et les enjeux régionaux.

Au printemps 2015, le gouvernement du Québec adoptait sous bâillon le projet de loi $28^{3}$. Cette loi entraîne des modifications à une cinquantaine de lois antérieures et aura donc des effets majeurs dans plusieurs secteurs socio-économiques au Québec, notamment en matière de gouvernance des forêts. La Conférence régionale des élus (CRÊ), considérée depuis sa création en 2004 comme linterlocutrice privilégiée du gouvernement en matière de développement régional (MRN 2013), était ainsi abolie dans chacune des régions. Les CRÉ étaient investies d'importantes responsabilités dans le domaine forestier, parmi lesquelles la mise sur pied et la coordination des CRRNT (p. ex. composition, règles de fonctionnement) qui étaient responsables de lanimation des Tables

\footnotetext{
${ }^{2} \mathrm{Au}$ Québec, la Loi sur l'aménagement durable du territoire forestier (L.R.Q., c. A-18.1), adoptée en mars 2010, accorde au ministre des Forêts, de la Faune et des Parcs la responsabilité de l'élaboration des plans d'aménagement forestier tactiques (PAFIT), révisés tous les cinq ans, et opérationnels (PAFIO), qui contiennent principalement les secteurs d'intervention où sont planifiées les activités de récolte et d’aménagement (MFFP 2016). Comme nous le verrons plus loin, ces plans sont soumis à la consultation publique.

${ }^{3}$ Le nom complet du projet de loi 28 est Loi concernant principalement la mise en ceuvre de certaines dispositions du discours sur le budget du 4 juin 2014 et visant le retour à l'équilibre budgétaire en 2015-2016.
}

GIRT, lorganisation des consultations autour des PAFI et du règlement des différends (voir Fig. 1). Dans le contexte de l'abolition des CRÉ, chacun des territoires se voit dans l'obligation de restructurer ses lieux de concertation et ses processus participatifs en matière de gestion forestière.

\section{But de la recherche}

Compte tenu du contexte d'incertitude actuel, le but de cette étude est d'explorer les répercussions qu'auront les changements induits par ladoption du projet de loi 28 - et plus particulièrement la disparition des CRÉ - sur la participation publique au sein des lieux de concertation propres au milieu forestier. Plusieurs auteurs ont abordé la question de la gouvernance forestière au cours des dernières années (p. ex. Andersson 2006, Howlett and Rayner 2006, Ribot et al. 2006, Larson and Soto 2008, Fréchette 2009, Chiasson et Leclerc 2013), alors que d’autres se sont intéressés aux limites de la participation publique dans un contexte forestier (p. ex. Buchy and Hoverman 2000, Martineau-Delisle and Nadeau 2010, Fortier et Wyatt 2014). Cependant, peu de chercheurs se sont attardés spécifiquement aux impacts de l'abolition de structures régionales sur les processus délibératifs associés à la gestion des forêts.

Dans le cadre de cette étude, nous nous sommes intéressés plus spécifiquement au point de vue des acteurs actifs au sein de deux structures de concertation qui touchent les forêts québécoises, soit les tables locales de gestion intégrée des ressources et du territoire (Tables GIRT) et les forums des Commissions régionales sur les ressources naturelles et le territoire (CRRNT). Avant d'aborder le contexte institutionnel de mise en place de ces lieux de concertation, nous allons dans un premier temps revenir sur la question de la participation publique dans la gestion des forêts. Après avoir explicité notre démarche méthodologique, nous présenterons et discuterons des résultats en faisant ressortir quelques éléments de convergence et de divergence entre les régions ciblées, ce qui nous permettra de proposer des pistes de recherche pour l'avenir.

\section{La participation publique dans la gestion des forêts}

La participation publique dans la gestion des ressources forestières est désormais largement répandue à travers le monde (Reed 2008, Johansson 2016). Dans son acception contemporaine, la participation publique peut être définie comme un processus volontaire au sein duquel des individus ou des groupes peuvent échanger de l'information, exprimer des opinions, définir des intérêts et potentiellement influencer les résultats d'un processus (ILO 2000). On considère à cet effet que le tournant délibératif en matière de gestion des ressources naturelles reflète le besoin de l'État de coopérer avec les acteurs de la société civile pour composer avec des questions environnementales complexes au sujet desquels persiste une incertitude (Parkins and Mitchell 2005, Bouthillier et Roberge 2007, Bäckstrand et al. 2010). Dans la gestion des forêts plus particulièrement, ce tournant révèle une remise en question de lautorité scientifique des experts de la foresterie par la société civile (Chiasson et al. 2006).

La participation à la gestion des ressources forestières peut être considérée autant comme une approche que comme un outil de gestion, selon quon adopte un point de vue principalement idéologique ou pragmatique, bien que ces deux catégories ne soient pas mutuellement exclusives (Buchy and 


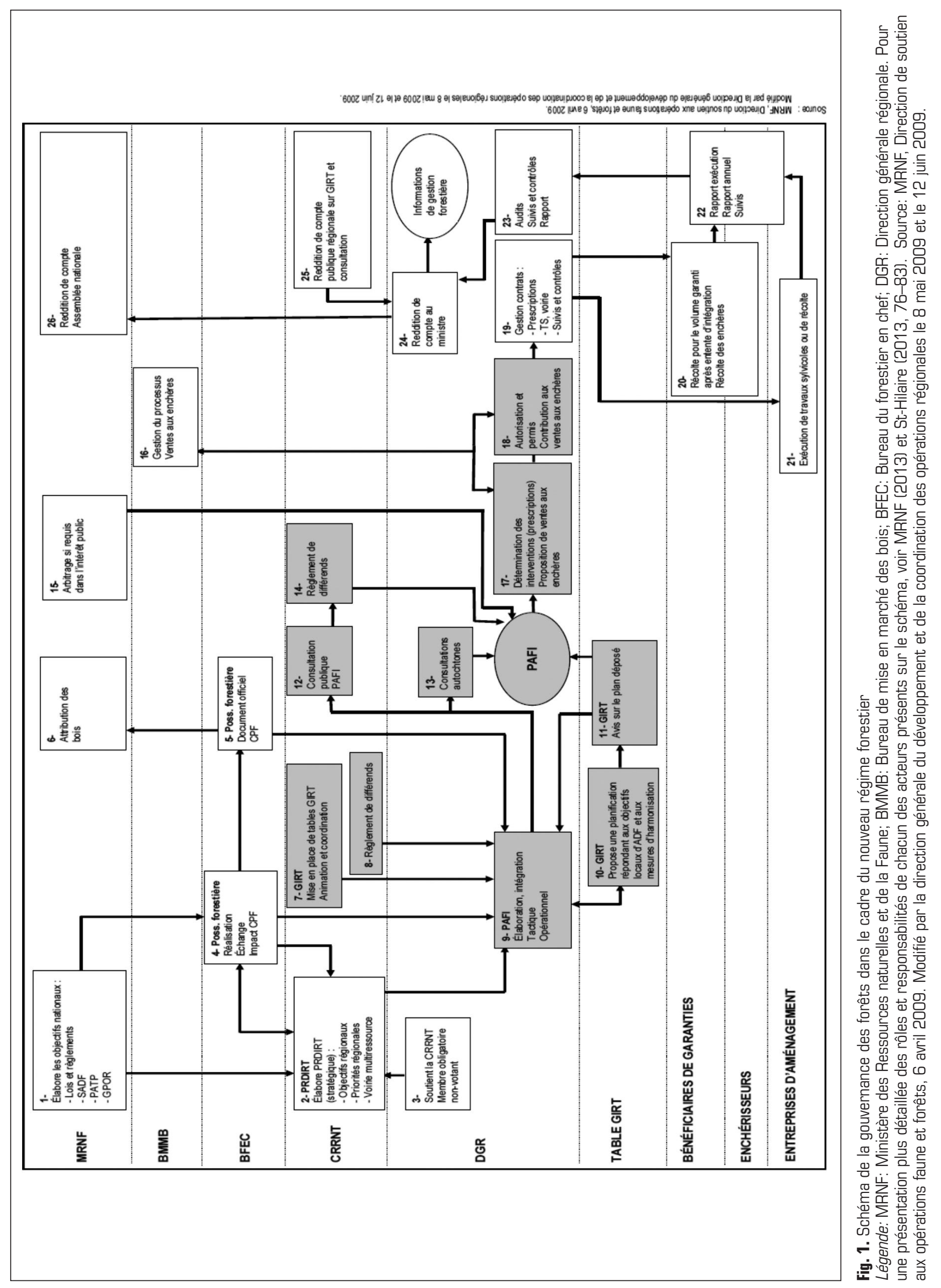


Hoverman 2000). Cette participation peut par ailleurs revêtir plusieurs formes que l'on situe généralement le long d'un continuum incluant l'échange d'information unidirectionnel, la consultation et la collaboration ou la cogestion, soit d'une participation indirecte à une participation directe (Beckley et al. 2005). Un autre continuum situe les approches participatives en fonction du type de participants représentés telles des assemblées publiques ouvertes à tous, des groupes de discussion restreints et des tables ou forums de participation pour les intervenants sectoriels ou les experts (Reed 2008, Martineau-Delisle 2013, Bruna-Garcia and Marey-Perez 2014).

Les études recensent plusieurs bénéfices liés à l'implication d'une diversité d'acteurs à la prise de décision en matière de gestion des ressources naturelles en territoire public. En plus dêtre une exigence démocratique, la participation publique est considérée, dans plusieurs contextes nationaux, comme une façon de refléter l'ensemble des intérêts et des valeurs sociales (Hilsop et al. 2004, Primmer and Kyllönen 2006). Une démarche de participation adéquate peut permettre d'améliorer la qualité des décisions prises et l'efficacité de leur mise en œuvre, tout en leur conférant une plus grande légitimité à long terme et en permettant d'atteindre des objectifs de foresterie durable (Howlett and Rayner 2006, Pappila and Pölönen 2012, Bruna-Garcia and Marey-Perez 2014). Le développement de relations de confiance entre les gestionnaires de la forêt et la population, ainsi que l'échange de connaissances qui en résulte peuvent atténuer les risques de conflits et réduire les délais des opérations forestières (Pappila 2013, Smith et al. 2013).

Malgré l'importance des recherches sur la question, il n'existe aucun consensus sur la meilleure façon de mettre en œuvre une démarche de participation publique qui permette une réelle gouvernance collaborative (Kangas et al. 2010). Cela est attribuable en grande partie aux multiples objectifs recherchés par la participation publique et au fait que les retombées ne sont généralement pas quantifiables (Lawrence and Daegan 2001). Rowe and Frewer (2000) identifient deux types de critères d'évaluation de la participation publique. Le premier porte sur la construction et la mise en œuvre d'une procédure de participation publique, tandis que l'autre touche à l'acceptation publique de la procédure elle-même. Le cadre proposé par Webler and Tuler (2001) paraît instructif à cet égard. Ces auteurs identifient quatre perspectives distinctes définissant une bonne participation: 1) la crédibilité et la légitimité du processus; 2) la compétence et l'information; 3) la délibération démocratique; 4) le dialogue constructif et le transfert de connaissances, voir l'apprentissage entre les participants (Kangas et al. 2010). Les deux premières portent sur le cadre de la participation, tandis que les deux autres touchent plutôt le processus introduit par la participation.

La structure choisie pour assurer la participation, ses objectifs spécifiques et surtout le choix des intervenants (stakeholders) seront déterminants pour assurer la qualité et les bénéfices d'une telle démarche (Reed et al. 2009). Pour évaluer plus spécifiquement la participation publique délibérative au sein de comités ou de forums relatifs aux questions forestières, des critères sont présentés par Beckley et al. (2005) qui notent l'importance d'une bonne représentation des intervenants, des rencontres facilitées par un professionnel, un mandat clair et le soutien d'un organisme clé, généralement le ministère responsable de la forêt. Un processus de cet ordre est généralement encadré et répond à des échéances, il a une portée définie, plusieurs stades itératifs et s’applique à un territoire forestier bien délimité. Dans une étude sur les comités consultatifs publics œuvrant dans le secteur forestier au Canada, Parkins et al. (2006) soulèvent un certain nombre de défis auxquels font face plusieurs de ces comités, notamment lobtention de renseignements à jour et diversifiés, une représentation adéquate du public et les processus de groupe associés à létablissement d'un consensus et à la prise de décision. Kangas et al. (2010) évoquent également l'importance de la transparence et le pouvoir d'influencer le processus et ses résultats. On spécifie également que l'atteinte d'un consensus ne peut être la seule mesure du succès d'une démarche de participation, qui doit pouvoir également être ouverte à des désaccords constructifs (Beckley et al. 2005).

\section{La participation publique dans la gestion des forêts au Québec} La participation publique dans la gestion des forêts au Québec est relativement récente et remonte à la Loi sur les forêts instituée en 1986. Dès 1987, la participation publique constitue une exigence légale qui va se mettre en place graduellement (Bouthillier et Roberge 2007, Martineau-Delisle and Nadeau 2010). Elle s'inscrit dans un mouvement pancanadien appuyé par la Stratégie nationale sur la forêt du Canada élaborée en 1992 et qui a exercé une importante influence sur les régimes forestiers des provinces en menant à la généralisation des procédures de participation publique (Lecompte et al. 2005, Chiasson et al. 2011). Alors quau départ les industriels, dépositaires de la planification forestière, soumettaient leur plan d'aménagement au public pour consultation, le régime forestier a progressivement introduit l'obligation de la concertation lors de la préparation de ces plans. À partie de 2001, l'article 54 de la Loi sur les forêts obligeait les industriels à inviter certains groupes afin de « prendre en considération les intérêts et préoccupations des autres utilisateurs du territoire de l'unité d'aménagement et de prévenir les différends concernant la réalisation des activités d'aménagement forestier " (Bouthillier et Roberge 2007, p. 811). Cette approche s'inspire des pratiques introduites par les organismes de certification forestière ayant réussi à faire reconnaître le principe de la participation publique (Beckley et al. 2005).

Avec la reconnaissance du rôle que joue la forêt dans le développement territorial, les politiques québécoises vont peu à peu tenter de favoriser la participation publique à la gouvernance forestière à l'échelle régionale. Plusieurs forums et initiatives ont vu le jour dans les régions du Québec, souvent selon les intérêts, la volonté et les ressources des acteurs territoriaux. La Loi sur l'aménagement durable du territoire forestier, adoptée le ler avril 2010 et entrée pleinement en vigueur en avril 2013, amène des changements importants dans la gestion des forêts publiques du Québec. L'adoption de cette loi a mené à la mise sur pieds de Tables GIRT dans l'ensemble des régions et est venue confirmer le rôle important des instances régionales dans la gouvernance des ressources forestières (voir Fig. 1).

Lobjectif des Tables GIRT est de « mettre en place une gestion participative dans lélaboration des plans d'aménagement forestier intégrés » (St-Hilaire 2013, p. 80). Le ministère des Ressources naturelles définit la GIRT du milieu forestier comme suit (Desrosiers et al. 2010, p. 5):

« [La GIRT est un] processus coopératif de gestion et de concertation. Ce processus réunit l'ensemble des acteurs et gestionnaires du milieu, porteurs d'intérêts collectifs publics 
ou privés, pour un territoire donné. Ce processus continu vise à intégrer, dès le début de la planification et tout au long de celle-ci, leur vision du développement du territoire, qui sappuie sur la conservation et la mise en valeur de l'ensemble des ressources et fonctions du milieu. »

Il ajoute que le succès de la GIRT repose sur la compréhension et le respect de cinq principes: 1) recherche de l'intérêt commun; 2) représentativité et concertation; 3) efficience dans les moyens d'action; 4) nécessité de résultat; et, 5) processus décisionnel démocratique.

Les tables de concertation ont été institutionnalisées au Québec en 2001, par la modification de la Loi sur les forêts (Chiasson et al. 2006, Leclerc et Andrew 2013). Une première génération des Tables GIRT avait été établie à partir du début des années 2000 dans certaines unités d'aménagement forestier, en Abitibi et en Outaouais notamment. Comme le rapportent Leclerc et Andrew (2013) dans leur étude longitudinale, malgré les objectifs initiaux, les rapports de force se sont avérés inégaux, les industriels et les représentants du Ministère ayant plus de ressources financières et de connaissances techniques en foresterie que les autres intervenants. Une des conclusions de l'étude est qu'il ne suffit pas de mettre en place une structure de concertation ouverte à une diversité d'acteurs pour en arriver à une gouvernance collaborative du secteur forestier; il faut aussi injecter des ressources pour faciliter la participation des acteurs plus marginalisés. Les auteurs soulignent également le rôle important que joue l'animateur de ces Tables, notamment afin de rééquilibrer les rapports de force entre acteurs.

En vertu de la Loi sur l'aménagement durable du territoire forestier (2010), la mise en place des Tables GIRT a été confiée aux CRÉ. Chaque CRÉ pouvait à son tour attribuer cette responsabilité au sein de sa structure, à une CRRNT (définie plus bas) ou, exceptionnellement, demander au ministre des Ressources naturelles et de la Faune de la confier à une municipalité régionale de comté (MRC) choisie de concert ${ }^{4}$. En confiant les Tables GIRT aux CRÉ, la nouvelle gestion se différencie de la précédente, notamment par l'unité territoriale régionale de leur gouvernance et «par la portée de leurs décisions, qui s'inscrit dans le continuum décisionnel de la nouvelle loi »(St-Hilaire 2013, p. 83). L'idée qui sous-tend les tables de concertation est la recherche d'un consensus acceptable pour l'ensemble des acteurs impliqués.

\section{Les Commissions sur les ressources naturelles et le territoire [CRRNT]}

Les Conférences régionales des élus (CRÉ) ont été mises en place par le gouvernement québécois en 2004 en remplacement des Conseils régionaux de développement (CRD) qui existaient dans certaines régions depuis les années 1970 (Belley 2008). Cette transformation confère davantage de pouvoir aux élus dans la mise en place de structures permettant la participation de la société civile à la gouvernance à l'échelle régio-

\footnotetext{
${ }^{4}$ Loi sur le ministère des Affaires municipales, des Régions et de l'Occupation du territoire, article 21.17.1, alinéa 3, introduit par l'article 308 de la Loi sur l'aménagement durable du territoire forestier. Au Québec, une MRC regroupe toutes les municipalités d'un même territoire d'appartenance formant une entité administrative qui est une municipalité au sens que l'entend la Loi sur l'organisation territoriale municipale (MAMOT 2016).
}

nale (Chiasson et Robitaille 2007). Les CRÉ se verront par la suite confier des responsabilités plus importantes en matière de gouvernance des ressources forestières. Dès décembre 2004, la Commission Coulombe avait recommandé la mise en place de commissions forestières régionales (CFR) relevant des CRÉ pour la gestion de la forêt publique (Coulombe et al. 2004). Par un nouveau décret en 2006, les CFR sont remplacées par les Commissions régionales sur les ressources naturelles et le territoire (CRRNT) relevant toujours des CRÉ et constituant les instances régionales de concertation sur ces questions (St-Hilaire 2013). Les modèles mis en place dans chacune des régions du Québec différaient quant à la structure, au fonctionnement et à l'administration (Bachand 2008). À travers les CRRNT, le gouvernement confiait ainsi aux CRÉ la tâche d'intégrer la gouvernance des différentes ressources naturelles dans ces initiatives de régionalisation.

Une des fonctions premières de la CRRNT, avant les changements institutionnels récents, était lélaboration du Plan régional de développement intégré des ressources et du territoire (PRDIRT) qui traduit les orientations et priorités régionales et permet l'allocation des fonds à des projets spécifiques. Les CRRNT étaient également en mesure de mettre en place des forums régionaux afin de documenter certains enjeux liés aux ressources naturelles et d'assurer la diffusion des connaissances en ce domaine, le plus souvent dans le but de répondre à une demande du milieu.

Cette étude, menée en temps réel dans la foulée de l'abolition des CRÉ, a un caractère rétrospectif et prospectif. Au regard de la spécificité et de l'historique de chacun des lieux de concertation mentionnés, elle a pour objectif d'examiner les perceptions des intervenants régionaux par rapport à deux choses: 1) la pertinence et l'efficacité de la participation publique au sein des structures en place jusquà l'abolition des CRÉ; et, 2) la résilience des modes de collaboration dans la gestion des forêts dans ce contexte de changement institutionnel. Par résilience, nous entendons la volonté et la capacité des intervenants à maintenir un certain niveau de concertation à léchelon régional.

\section{La Méthodologie}

Considérant la période d'incertitude et de transition dans laquelle s'inscrit cette étude, nous avons adopté une approche qualitative exploratoire (Trudel et al. 2007). Pour ce faire, nous avons ciblé quatre régions du Québec qui correspondent à des réalités sociopolitique, économique et environnementale différentes (voir tableau 1). Alors que la Gaspésie et la Mauricie peuvent être considérées comme des " régions ressources », c'est-à-dire des territoires organisés en fonction des industries liées aux ressources naturelles, la région de la Capitale-Nationale subit l'influence directe d'un grand centre urbain (la Ville de Québec). Par ailleurs, bien qu’au Québec la forêt productive et accessible, du point de vue de la production commerciale de bois, soit largement publique, la proportion de forêt publique par rapport à la forêt privée diffère d'une région à l'autre. Ainsi, le territoire forestier de l'Estrie est à plus de $90 \%$ sous tenure privée, alors que cette proportion est de $31 \%$ ou moins pour les trois autres régions.

Pour la collecte de données, nous avons principalement fait appel aux entretiens semi-directifs auprès d'acteurs clés. Avec l'entretien semi-directif, le chercheur dispose d'un certain nombre de thèmes et de questions organisés dans un 


\begin{tabular}{|c|c|c|c|c|c|c|}
\hline Région & Superficie $\left(\mathrm{km}^{2}\right)$ & Population (hab.) & $\begin{array}{l}\text { Densité de } \\
\text { population } \\
\left(\text { hab./ } / \mathbf{k m}^{2}\right)\end{array}$ & $\begin{array}{l}\text { Terrains forestiers } \\
\text { productifs } \\
\text { accessibles }\left(\mathbf{k m}^{2}\right)\end{array}$ & $\begin{array}{c}\text { Volume de bois } \\
\text { récolté en forêt } \\
\text { publique et } \\
\text { privée }\left(\mathbf{k m}^{3}\right)\end{array}$ & $\begin{array}{c}\text { Proportion de } \\
\text { forêt privée } \\
\text { productive }(\%)\end{array}$ \\
\hline Estrie & 10456 & 292021 & 27,9 & 7668 & 1046,8 & 91 \\
\hline Gaspésie & 20474 & 97910 & 4,8 & 16382 & 1362,8 & 18 \\
\hline Mauricie & 39907 & 267916 & 6,7 & 31343 & 2927,9 & 17 \\
\hline Capitale-Nationale & 19798 & 646800 & 32,7 & 15657 & 992,7 & 31 \\
\hline
\end{tabular}

Source : a) Fédération des producteurs forestiers du Québec, édition 2015 ; b) Ressources et industries forestières, Portrait statistique, édition 2015 , MRNFP.

Tableau 2. Nombre d'entretiens par catégorie d'acteurs

\begin{tabular}{|c|c|c|c|c|c|c|c|}
\hline Région & $\begin{array}{c}\text { Acteurs } \\
\text { municipaux/ } \\
\text { MRC }\end{array}$ & $\begin{array}{c}\text { Ministère/ } \\
\text { institutions du } \\
\text { gouvernement }\end{array}$ & $\begin{array}{l}\text { Représentants } \\
\text { de la forêt } \\
\text { privée }\end{array}$ & $\begin{array}{c}\text { Personnel } \\
\text { des CRÉ }\end{array}$ & $\begin{array}{l}\text { Industriels } \\
\text { forestiers }\end{array}$ & $\begin{array}{l}\text { ONG / autres } \\
\text { utilisateurs } \\
\text { de la forêt }\end{array}$ & Total \\
\hline Estrie & 1 & 0 & 1 & 2 & 1 & 2 & 7 \\
\hline Gaspésie & 2 & 2 & 2 & 3 & 1 & 4 & 14 \\
\hline Mauricie & 2 & 2 & 0 & 1 & 0 & 2 & 7 \\
\hline Capitale-Nationale & 1 & 1 & 3 & 2 & 0 & 2 & 9 \\
\hline Total & 6 & 5 & 6 & 8 & 2 & 10 & 37 \\
\hline
\end{tabular}

canevas qui va orienter l'entretien (Hoggart et al. 2002). L'utilisation d'un tel outil permet la collecte d'informations similaires auprès de chacun des acteurs et facilite l'analyse ultérieure des données. Bien que nous l'ayons modifié quelque peu afin de refléter les caractéristiques des différents groupes d'acteurs, les thèmes suivants ont été abordés à travers un certain nombre de questions: organisation et rôle du répondant; historique et structure des lieux de concertation; participation et dynamique de concertation; facteurs ayant facilité ou inhibé la concertation; compétences et représentativité des acteurs régionaux; impacts de l'abolition des CRÉ sur la concertation; avenir de la concertation régionale. Les questions ne sont pas nécessairement posées dans un ordre précis et certaines peuvent s'ajouter en cours de route. On parle alors de la récursivité de l'entretien, c'est-à-dire qu'on se sert de ce qui a été dit pour produire de nouvelles questions pertinentes à la recherche (Coulombe et al. 2004). Le chercheur et l'enquêté disposent donc d'un espace de liberté; le rôle du chercheur est alors de recentrer l'entretien quand le répondant sécarte trop de ses priorités.

Nous avons choisi les personnes à interroger par échantillonnage dirigé, c'est-à-dire en ciblant des intervenants ayant participé activement à la concertation régionale en matière de forêt au cours des dernières années. Ceux-ci avaient donc une connaissance approfondie de la participation publique dans la gestion des forêts, laquelle ils pouvaient situer dans une perspective historique. Un total de 37 entretiens (45 à 120 minutes; enregistrés) ont été réalisés en personne entre avril et octobre 2015 par deux des co-chercheurs (voir tableau 2). Étant donné le caractère exploratoire de la recherche, nous navons pas cherché la représentativité. Ainsi, tous les grands groupes d'acteurs ne sont pas représentés de façon uniforme dans chacune des régions. En ce qui concerne les industriels forestiers par exemple, qui sont sousreprésentés, ils se sont avérés plus difficile à intéresser à la recherche, certains invoquant le manque de temps pour ce genre de contribution. Par ailleurs, bien quétant parfois présents aux tables de concertation dans certaines régions, les représentants des communautés autochtones nont pas été contactés compte tenu des limites inhérentes à la recherche (p. ex. contraintes temporelles et financières). Ceci représente une limite importante à la recherche et il est nécessaire d'en tenir compte lors de l'interprétation des résultats. Afin de protéger la confidentialité des intervenants, nous avons assigné des codes à chacun d'eux.

De façon à mieux contextualiser les propos des intervenants, nous avons également fait appel à l'analyse documentaire (p. ex. projet de loi $n^{\circ} 28$ et autres documents législatifs pertinents, documents produits par les CRÉ, rapports, procès verbaux). Ces documents ont été analysés à partir des mêmes thèmes que les entretiens, mais de façon plus souple, l'objectif étant plutôt de compléter ou confirmer certaines informations colligées lors des entretiens. Nous avons finalement participé à quelques évènements qui s'inscrivaient dans les activités de concertation régionale ( $\mathrm{p}$. ex. rencontres régionales des Tables GIRT, Forum sur la production de bois et l’aménagement durable).

Par rapport aux objectifs de la recherche et à la particularité de la collecte de données, l'analyse qualitative de contenu est la méthode que nous avons retenue pour analyser les divers matériaux recueillis sur le terrain (Hsieh and Shannon 2005, Elo and Kyngäs 2007, Zhang and Wildemuth 2009). Dans le choix des méthodes d'analyse, nous avons opté pour une approche mixte qui fait à la fois appel à la déduction et à l'induction. Bien que certains auteurs insistent sur l'une ou l'autre approche, nous allons plutôt dans le sens de Cloke et al. 
(2004, p. 216) pour qui « such distinctions are invariably overdrawn and [...] most research tends to contain both inductive and deductive moments in a constant, if uneven and sometimes unappreciated, dialogue. " La forme d'analyse de contenu utilisée ici correspond à ce que Hsieh and Shannon (2005) appellent l'analyse de contenu dirigée, c'est-à-dire que lon part de catégories dérivées de la théorie pour coder, mais on laisse tout de même place à lémergence afin de revoir la proposition théorique ou du moins y apporter quelques nuances.

Les entretiens ont été transcrits verbatim puis codés à la main par deux des co-chercheurs afin de faire ressortir un certain nombre de patterns du corpus. Saldana définit un code en recherche qualitative comme étant généralement un mot ou une courte phrase qui assigne symboliquement un attribut important, évocateur ou qui résume de façon succincte une portion de texte ou de données visuelles. Cest une construction générée par le chercheur qui associe un sens à chacune des données (une phrase, un paragraphe) pour plus tard s'en servir pour détecter des patterns, créer des catégories, raffiner la théorie ou accomplir d'autres processus analytiques (Ibid.). Afin d'assurer une certaine uniformité dans l'utilisation des codes, les co-chercheurs ont d'abord codé les deux mêmes entretiens choisis au hasard, puis ils les ont comparés et en ont discuté. Le reste des entretiens ont ensuite été partagés en deux selon les régions. Au final, nous avons regroupé la vingtaine de codes et sous-codes obtenus en deux grandes catégories, soit: 1) participation publique et dynamique de concertation régionale (avant l'abolition des CRÉ); et, 2) résilience des lieux de concertation (voir annexe 1). Cette démarche nous a servi à structurer les résultats présentés à la section suivante.

\section{Résultats}

Participation publique et dynamique de concertation régionale Cette première partie sattarde principalement aux lieux de concertation (Tables GIRT et forums chapeautés par la CRRNT) avant la fermeture des CRÉ afin de mettre en contexte le point de vue des intervenants quant à l'avenir de ces lieux. Les modèles des CRRNT et les dossiers traités différaient de façon importante d'une région à l'autre, ce qui limite toute forme de généralisation sur le fonctionnement de cette instance. Cependant, la CRRNT aurait eu une importante autonomie dans l'animation de forums régionaux ou de tables sectorielles sur les forêts. Les avis convergent quant à l'importance des forums régionaux au sein des CRRNT. En Gaspésie par exemple, la CRRNT animait la Table Forêt, une structure originale née d'une demande du milieu forestier (G-8, 11, $13)^{5}$. Cette table a créé différents comités, formés des intervenants membres, afin de travailler sur des dossiers précis tels la certification forestière ou l'accès au territoire public. Dans la région de Québec, la Table Forêt de la CRRNT aurait répondu à un besoin. « Pour qu'il y ait une Table Forêt, il faut qu'il y ait une demande de la base et une volonté des élus municipaux. Dans la Capitale-Nationale, je pense que les deux étaient là » (QC-2). À travers leur participation à la Table Forêt, les acteurs pouvaient ainsi suivre les dossiers en cours, prendre

\footnotetext{
${ }^{5}$ La lettre correspond à la région (G: Gaspésie; QC: CapitaleNationale; MC: Mauricie; E: Estrie) et le chiffre à un acteur en particulier.
}

connaissance de ce qui se déroulait au sein des autres organismes et prendre contact avec les acteurs politiques, notamment les MRC. Tout cela permettait d’améliorer la synergie entre les acteurs et d’arriver plus facilement à des terrains dentente. En somme, en raison de son accent sur les enjeux régionaux et des relations qui se développent entre les acteurs, la plupart des acteurs interrogés dans chacune des régions (29/37) accordaient une grande importance à la CRRNT comme lieu de concertation.

Par ailleurs, le fonctionnement des Tables GIRT, dans la mesure où il est encadré par la loi, ne bénéficie pas de la même autonomie que les CRRNT. Les Tables GIRT sont plutôt opérationnelles et sont associées à un territoire spécifique (c.-à-d. une unité d’aménagement forestier), tandis que les forums régionaux et les tables sectorielles sur les forêts traitent d’enjeux à portée régionale. «La recette Table GIRT, cétait très officiel, avec beaucoup de contraintes, ce qui fait que ce nétait pas toujours adapté à ce que les gens auraient voulu » (G-14). Malgré cela, certains insistent sur la pertinence de la Table GIRT et de son échelle d'intervention, dans les régions de la Capitale-Nationale et de la Gaspésie notamment (QC-5, 7; G-2, 4, 5). Malgré le caractère opérationnel des Table GIRT, la participation à celles-ci est décrite comme une démarche de partage de connaissances et de conscientisation, au même titre que les forums régionaux. " Les Tables GIRT viennent briser la glace entre le milieu forestier et le milieu municipal et régional » (QC-7). Létablissement d'un dialogue permet aux divers acteurs présents en ces lieux de concertation de mieux saisir les enjeux auxquels sont confrontés les autres acteurs forestiers traditionnels, notamment les industriels et le ministère des Forêts, de la Faune et des Parcs (MFFP) (ciaprès appelé Ministère) (p. ex. G-3, 9; E-7). Pour l'industrie notamment, les Tables GIRT sont pertinentes en ce quelles permettent de désamorcer les conflits d'usage (G-5).

Cependant, des intervenants ont perçu une redondance entre les lieux de concertation, notamment entre les Tables GIRT de chaque unité d'aménagement forestier d'une même région où on retrouve souvent les mêmes acteurs (p. ex. G-1). D’autres ont insisté sur la synergie qui existait entre les lieux de concertation - Tables GIRT et forums régionaux. Par exemple, plusieurs aspects forestiers importants nétaient pas traités aux Tables GIRT en Gaspésie, comme laccès global au territoire, les insectes ravageurs ou les feux de forêts. La Table Forêt de la CRRNT venait combler ces lacunes. Par ailleurs, pour certains intervenants de la région de la Capitale-Nationale, notamment ceux de la forêt privée, la CRRNT était perçue comme une structure redondante par rapport à ce qui existait déjà dans la région $(\mathrm{QC}-1,3)$. De plus, pour les petits organismes, leurs ressources humaines et financières étant limitées, il leur était plus difficile de participer aux multiples instances de concertation (E-5).

\section{Compétences et représentativité}

Parmi les éléments qui semblent avoir grandement contribué au succès de certains lieux de concertation figurent les qualités personnelles et les compétences des animateurs et des participants en général. Plusieurs ont souligné que la compréhension inégale des enjeux de la part des divers acteurs venait affecter la dynamique de concertation (G-2, 14; MC-3, 4, 5). Certaine personnes pouvaient se sentir dépassées lorsque la discussion devenait plus technique et pointue. Malgré tout, la 
majorité des intervenants rencontrés (25/37) s'entendent à l'effet que la plupart des secteurs étaient représentés dans les divers lieux de concertation et étaient en mesure de défendre leurs intérêts, autant à la CRRNT quaux Tables GIRT. En Gaspésie par exemple, la CRÉ a eu une vision inclusive pour les Tables GIRT et ne s'est pas arrêtée aux organisations obligatoires, ce qui aurait favorisé la diversité (G-2). Des acteurs ont donc été invités à se joindre aux Tables GIRT en cours de route lorsque les participants réalisaient que leur présence était pertinente pour faire évoluer la discussion. Cependant, selon les données recueillies, les grandes absentes de ces lieux de concertation sont les communautés autochtones, une situation qui se répète dans la plupart des régions du Québec (E-2; QC-6; MC-5). D’après les intervenants qui se sont prononcés sur cette question, cela s'explique en grande partie par le fait que bon nombre de ces communautés ont des revendications territoriales en cours et se considèrent comme une nation à part entière.

De plus, dans la plupart des régions, les Tables GIRT étaient animées par la CRRNT, mais le rôle que jouait le Ministère était très important, trop aux yeux de certains. En Gaspésie par exemple, le Ministère aurait été trop directif jusqu'ici (G-2, 9, 14). "Côté coordination, nous étions beaucoup contraints par les façons de faire du Ministère, qui sont provinciales. Ils ont des canevas qu'il faut suivre, des façons de consulter, des structures de table qui sont uniformes » (G-14). Parfois, les acteurs parvenaient à un consensus qui ne semblait pas satisfaire le Ministère. «J'ai vu des consensus avec des industriels forestiers, tout le monde à la table, puis le Ministère dire: non, on ne peut pas faire ça » (G-9). De plus, au plan logistique, les journées étaient très chargées, limitant la capacité des membres d'analyser l'information et de prendre de bonnes décisions $(G-1,6)$. Des interrogations similaires soulevées dans les cas de Québec et de la Mauricie expliquent le désintérêt de plusieurs intervenants pour les Tables GIRT. "Les organismes qui avaient beaucoup d'attentes ont vu que le Ministère ne leur donnait pas ou ne considérait pas lensemble de leurs revendications ou de leurs demandes. Cela a créé beaucoup de frustration [et] les gens se sont désintéressés » (MC-5). À cet égard, plusieurs critiquent les limites de la concertation avec les représentants du Ministère, ce qui en incite certains à percevoir la Table GIRT comme une instance largement instrumentalisée au profit de la réalisation des plans d’aménagement forestier intégré $(\mathrm{QC}-4,6)$.

\section{Un outil mobilisateur: le Plan régional de développement intégré des ressources et du territoire (PRDIRT)}

En Gaspésie et en Estrie, la réalisation du PRDIRT semble avoir joué un rôle clé dans la mobilisation des acteurs et la mise en place d'un dialogue autour des enjeux forestiers. Bien que cet exercice ait demandé beaucoup d'investissement en argent et en temps de la part des divers intervenants, le PRDIRT était perçu par plusieurs comme un outil efficace pour assurer le développement sur l'ensemble de la région et maintenir une vision concertée de ce développement. "Je pense que [lintérêt pour la concertation au sein de la CRRNT] est allé croissant, puis un des éléments qui a fait que le momentum sest maintenu, c'est la conception du plan de développement régional. Cétait un outil concret [et] il y avait de l'argent associé à ça au bout » (E-1). Suite à l'annonce de labolition des CRÉ, plusieurs intervenants ont déploré le fait que les ressources investies dans le PRDIRT pourraient avoir été en partie gaspillées puisque largent et les structures de concertation pour la mise en œuvre ont disparues $(G 9,10)$ "Que va-t-il arriver de ce plan? Je ne veux pas y penser, je me dis que ça na pas de bon sang! Ça a coûté des millions! » (G9). Néanmoins, dans la région de Québec notamment, il semble que la mobilisation nait pu être maintenue dans le temps, et ce pour un ensemble de facteurs, dont la diminution des ressources disponibles, ou la fin de la réalisation du PRDIRT (QC-6, 8). Dans cette région, la pertinence du PRDIRT était remise en cause au même titre que la CRRNT elle-même (QC-2, 7).

\section{Résilience des lieux de concertation Impacts de l'abolition des CRÉ}

La transition imposée par l'abolition des CRÉ est perçue différemment dans chacune des régions du Québec. L'opposition perçue entre les régions dotées d'un grand centre urbain et les régions rurales ressort ici, telle quexprimée par exemple par un intervenant de la Gaspésie. "Il y a des régions où [la transition] est beaucoup plus facile [...] Pour Québec et Montréal, cest facile, ils ont de l'argent en masse! [...] Tandis qu'ici, les enjeux sont beaucoup plus fondamentaux, beaucoup plus terre à terre » (G-8). L'abolition des CRÉ et des structures de concertation en matière de ressources naturelles laisse un goût amer à plusieurs intervenants qui expriment incertitude et inquiétude (G-2, 4, 9, 12; E-1; MC-5; QC-1). Il y a une crainte que les enjeux régionaux soient quelque peu abandonnés, qu'il y ait une démobilisation des intervenants régionaux. En Mauricie par exemple, cette incertitude fait redouter le pire en matière de développement économique étant donné le délai dans la mise en place de nouvelles structures (MC-5).

Certains perçoivent les récentes décisions du gouvernement comme un acte de recentralisation à léchelle du Québec (p. ex. E-1). Même au sein du Ministère, certaines personnes n'hésitent pas à critiquer à la fois la décision du gouvernement d'abolir les structures de concertation régionales, mais aussi de tendre vers une recentralisation plus générale des pouvoirs. " J'ai de la difficulté avec le cadre gouvernemental, jai de la difficulté avec Québec. Je suis donc très à l'aise avec ce système de régionalisation, de "décentralisation" qui nous permettait de moduler nos activités en fonction des réalités régionales. [...] Cest clair que ça sest perdu [avec la loi 28]» $(\mathrm{X}-2)^{6}$. D'autres intervenants ont soulevé l'idée que la disparition des CRÉ compromet la participation du public à la gouvernance des ressources à léchelle régionale. « Le Ministère va juste être content [de la disparition de la CRÉ], parce que pour lui, c'est pas mal moins complexe. Il n'aime pas devoir changer sa planification à cause des préoccupations, par exemple » (E-2). Toutefois, pour d’autres intervenants, la CRÉ représentait une centralisation à léchelle régionale qui nétait pas toujours stratégique dans la gouvernance des forêts, puisque certaines régions, comme celle de Québec, englobent différents biomes forestiers (MC-2; QC-4). Même parmi ceux qui sont plutôt favorables à une révision de la façon de faire la concertation en région, certains questionnent la manière dont les décisions ont été prises et imposées (G-14).

\footnotetext{
${ }^{6}$ Nous avons choisi ici de ne pas spécifier la région de l'intervenant afin d'éviter qu'il puisse être identifié.
} 


\section{Volonté et capacité des MRC à assumer la concertation}

Bien que cela varie quelque peu entre les régions et les MRC, la plupart des intervenants doutent de la capacité et de la volonté des élus et des MRC à prendre le relai en matière de concertation régionale. Plusieurs avancent que la prise en charge des divers dossiers qui touchent la gestion et la gouvernance des forêts commande des compétences particulières que bon nombre de municipalités ou de MRC ne possèdent pas pour l'instant. « Les élus et les MRC ne sont pas du tout outillés pour reprendre les dossiers des ressources naturelles. Jamais ils ne se penchaient là-dessus. On rencontrait les aménagistes des MRC périodiquement; pour eux, on [les professionnels des CRÉ] était la référence dans le dossier » (E-2).

À cela s'ajoute le fait qu'il y a maintenant moins d'argent disponible pour la concertation et le développement régional, puisque l'abolition des CRÉ a été faite dans un objectif de contrôle des dépenses gouvernementales (G-8). Les MRC disposent maintenant d'un montant général pour la coordination des Tables GIRT, les consultations publiques et la gestion des fonds du Programme d’aménagement durable des forêts, mais ce montant semble insuffisant pour inciter les MRC à en mettre une partie à disposition de projets à portée régionale (E-3). On redoute ainsi le retour en force d'une certaine forme de chauvinisme local ou ce que plusieurs ont appelé "lesprit de clocher", notamment en Gaspésie (G-4, 10). La CRÉ aurait permis d'estomper ce phénomène, en Gaspésie du moins, car les élus navaient alors pas le choix de travailler ensemble sur divers dossiers régionaux et dans l'intérêt de la région plutôt que pour leur seule municipalité ou MRC. Une personne bien au fait des enjeux (G-13) affirme que le danger avec le transfert des responsabilités aux élus est qu'il y ait une surpolitisation des enjeux de développement régional et de gouvernance des forêts. On craint par ailleurs une démobilisation et un désintéressement de la part des acteurs régionaux. «On y a crû à la GIRT, on l'a amené à un certain niveau, imparfait mais intéressant. Je me demande si on aura encore cette drive-là avec les MRC » (G-2). Bien que ce ne soit pas attribuable à la réorganisation régionale en cours, le fait que les élus changent régulièrement, au gré des élections, fait aussi craindre une certaine instabilité des structures de concertation et des priorités. Plusieurs personnes reprochent aussi le manque de vision de certains élus et le manque de continuité dans celle-ci (G-13; E-4; QC-8).

Malgré les nombreuses réticences évoquées précédemment, quelques intervenants pensent que les MRC sont un bon palier pour reprendre les responsabilités en matière de forêt et de concertation, surtout en ce qui a trait à la gestion de la Table GIRT qui est mieux encadrée et dont les objectifs sont plus spécifiques. Un participant gouvernemental (G-1) nous a indiqué que le transfert de gestion aux MRC ne devrait pas poser de problème majeur étant donné que se sont les mêmes acteurs qui devraient participer. « La CRÉ était mandatée pour gérer la table GIRT. C'est la table GIRT qui marche. Moi, que ce soit une MRC ou je ne sais trop qui va la gérer, ta table GIRT devrait continuer de vivre » (G-5). Pour un intervenant du secteur municipal de Mauricie qui a connu la période avant les CRÉ, cela signifie un retour aux anciens modes de fonctionnement, qu'il qualifie tout de même comme adéquats (MC-5).

\section{Avenues envisagées par les acteurs du milieu}

Depuis lannonce de la fermeture des Conférences régionales des élus (CRÉ) et la disparition des lieux et structures de concertation, plusieurs initiatives ont été prises par les acteurs du milieu afin de conserver certains acquis. Une stratégie à court terme, mise en œuvre dans deux des quatre régions, est l'incorporation de la CRRNT. En Gaspésie, lidée était de se préparer à toute éventualité dans ce climat d'incertitude. On voulait qu'il y ait une organisation en place au cas où des mandats pourraient lui être confiés rapidement. En Estrie, les acteurs ont entrepris des démarches pour incorporer la CRRNT, mais l'incorporation n'a pas été finalisée, notamment parce que le gouvernement a clairement mentionné qu'il n'allait pas reconnaître une telle structure (E-3). Quelques élus avaient travaillé assidument dans cet objectif, mais leur énergie se tourne plutôt vers une nouvelle structure de concertation plus ciblée, le Comité de production de bois. Cette initiative tente de répondre à un enjeu important dans la région où la forêt est de tenure privée à plus de $90 \%$, à savoir l'incapacité à favoriser l'exploitation forestière chez les propriétaires privés, ce qui amplifie le problème d’approvisionnement de certaines usines (E-1, 3). En Mauricie, un organisme incorporé serait déjà désigné pour prendre en charge les fonctions de la CRRNT (MC-1). Dans la région de la Capitale-Nationale, la Table des préfets a été désignée pour reprendre en charge certaines fonctions de concertation et déterminer les rôles des MRC de la région (QC-7). Cependant, certaines incertitudes persistent, tant en ce qui concerne la capacité que lacceptabilité du nouveau contexte institutionnel de coordination des Tables GIRT et, plus largement, de la concertation en matière de gestion forestière dans cette région.

\section{Discussion}

Cette étude exploratoire rend compte de trois constats concernant les perceptions sur la participation répandues parmi les intervenants rencontrés dans la gestion des forêts : 1) la reconnaissance de la pertinence du processus de participation publique et de la concertation à léchelle régionale pour la gouvernance des forêts; 2) une rupture dans la dynamique de délibération démocratique et du transfert de connaissance à cause de la prise en charge du processus par les acteurs municipaux; et, 3) l'impression d’ambiguïté que laissent les orientations et les décisions gouvernementales pendant la période de transition.

Au cours des 30 dernières années, soit depuis l'adoption de la Loi sur les forêts en 1986, le gouvernement québécois semble avoir résolument pris le tournant délibératif en matière de gestion des forêts (Parkins et Mitchell 2005, Bouthillier et Roberge 2007). Graduellement et de façon plus soutenue au cours des 10 dernières années, des modes de participation et de concertation en matière de gestion des territoires forestiers se sont mis en place dans les différentes régions étudiées, que ce soit à travers les Tables GIRT ou encore les forums animés par la CRRNT. L'abolition des CRÉ dans chacune des régions du Québec, à travers l'adoption de la loi 28 en avril 2015, représente donc en quelque sorte un point de rupture qui implique une réorganisation pour plusieurs intervenants du milieu forestier. Ce sentiment est d'autant plus fort dans les régions éloignées des grands centres, comme la Gas- 
pésie, où les capacités et les ressources pour se réorganiser sont plutôt limitées. Il importe cependant de faire une distinction entre les Tables GIRT et les forums régionaux.

En fonction du premier objectif de létude, soit d'examiner les perceptions de la qualité de la participation publique, les avis recueillis portent sur chacune des structures. Dans un premier temps, la CRRNT correspondait davantage à un forum délibératif permettant la collaboration d'experts sectoriels dans un exercice de participation directe (Bruna-Garcia and Marey-Perez 2014). À cet effet, une majorité de participants reconnait la pertinence de cette instance. Dans les quatre régions à létude, la CRRNT a procuré un forum ouvert aux enjeux du milieu en matière de gouvernance forestière, et des moyens afin de documenter les enjeux prioritaires pour éclairer les décisions à léchelle régionale. La CRRNT était généralement bien arrimée aux cadres règlementaires, programmes et initiatives préexistants, et permettait au mieux de les appuyer. Dans l'ensemble, les CRRNT apparaissent comme des structures qui favorisaient le développement de relations informelles et une meilleure connaissance des intervenants du milieu. Cependant, on observe dans certaines démarches de la CRRNT une absence dobjectifs clairs et de processus itératifs avec des échéances définies, ce qui a entraîné la démobilisation des intervenants au fil du temps dans certaines régions. Les limites dans l'opérationnalisation du PRDIRT décriées par certains rendent compte de ces difficultés. De plus, l'importance de l'investissement en temps lié aux exigences d'un processus délibératif ouvert incite plusieurs à affirmer que des réformes auraient pu être apportées, sans pour autant mettre fin à la démarche.

La prise en charge des Tables GIRT par la CRRNT aurait favorisé une harmonisation des procédures au sein d'une région et la transversalité dans la prise en considération de certains enjeux, lorsque nécessaire. De plus, il semble y avoir eu une certaine ambiguïté sur les finalités de cette démarche de concertation : elle était certes coordonnée par les membres de la CRRNT, mais perçue par plusieurs comme un instrument de consultation afin de permettre aux experts du Ministère de recueillir les avis et préoccupations des utilisateurs de la forêt, pour procéder à la planification des activités forestières. Ce qui pour autant naurait rien changé, selon certains, au pouvoir démesuré qui ultimement revient au Ministère comme gestionnaire de la forêt publique et responsable de l'encadrement des bénéficiaires de garanties d'approvisionnement. Ainsi, l'abolition des CRÉ concernant la coordination des Tables GIRT est interprétée comme favorisant soit le statu quo ou même une augmentation potentielle du pouvoir du Ministère.

En ce qui a trait au deuxième objectif, le maintien des Table GIRT pourra assurer une certaine continuité, et une forme de résilience dans les processus de participation publique. En effet, les Tables GIRT sont actuellement inscrites dans la Loi sur laménagement durable du territoire forestier et font donc partie intégrante du nouveau contexte de gouvernance forestier au Québec, malgré l'abolition des CRRNT qui en avaient la responsabilité. Bien que la Table GIRT introduise certaines formes de collaboration qui permettent une participation directe, sa portée demeure toutefois limitée par les balises de la planification forestière. Ainsi, plusieurs intervenants perçoivent les Tables GIRT davantage comme un mode de consultation que de collaboration (au sens où l'entend Beckley et al. 2005). Les critiques mettent parfois en cause la coordination effectuée par la CRRNT, notamment en ce qui concerne la gestion des discussions et le manque d'expertise de l'animateur, ou même le manque d'appui des experts du Ministère. Bien que la représentation des utilisateurs de la forêt ait été adéquate, de l'avis du plus grand nombre, lintégration des enjeux et des préoccupations des utilisateurs de la forêt aurait été variable. Les intervenants semblent, en majorité, reconnaitre la pertinence de ce lieu de concertation, mais insister sur sa perfectibilité, ou sur la définition de ses objectifs, qui dans sa forme actuelle aurait créé beaucoup d'attentes déçues. Pour plusieurs, l'abolition des CRÉ est perçue comme une opportunité afin d'apporter certains changements au fonctionnement des Tables GIRT.

Avec l'adoption de la loi 28 et l'abolition des CRÉ, la gestion, lorganisation et la portée des Tables GIRT est présentement revue dans chacune des régions. Dans la plupart des cas, la coordination des Tables GIRT et des consultations publiques se voit confiée à une ou plusieurs MRC, désignées de concert par les préfets d'une région, comme cela avait été prévu par la loi. C'est aux MRC désignées qu'incombe la tâche de définir le modèle de concertation approprié, notamment pour se conformer au Programme d'aménagement durable des forêts (PADF) annoncé en juillet 2015 (Harvey 2015).

En fonction du cadre proposé par Webler and Tuler 2001, (voir aussi Kangas et al. 2010), nous sommes en mesure de mieux saisir le point de vue des participants. Il semble ainsi que la perception des modes de participation publique dépende de la façon dont chaque intervenant évalue la pertinence des structures qui demeurent, soit la Table GIRT et les autres exigences du PADF prises en charge par les MRC ( $\mathrm{p}$. ex. les consultations publiques au sujet du plan d'aménagement). En effet, certains évaluent la participation publique à laune du cadre (crédibilité et légitimité du processus), alors que d'autres s'intéressent plutôt au processus lui-même (délibération, dialogue). Pour reprendre les propos de Buchy and Hoverman (2000), on observe donc que la participation publique à la gestion des forêts au Québec relève à la fois d'une approche (importance d'intégrer le point de vue de divers acteurs) et d'un outil de gestion (comme en font foi les Tables GIRT). Les différences de point de vue peuvent en partie s'expliquer par les attentes des acteurs rencontrés en ce sens. Alors que le Ministère va plutôt mettre l'accent sur la concertation comme outil de gestion, d'autres acteurs, notamment ceux de la société civile, voient ces lieux de concertation comme dexcellentes plateformes de délibération démocratique.

Quoi qu'il en soit, malgré les divergences d'opinion, l'abolition des CRÉ constitue un rétrécissement des canaux de communication et de rétroaction entre différents intervenants. La CRRNT dans plusieurs régions avait même été appropriée par la société civile régionale, lui permettant de se faire entendre et de se faire connaître, dans lespoir d'influencer les décisions politiques régionales (Lévesque 2004). Ils permettent par ailleurs aux gens présents de prendre conscience de la réalité des autres, processus qui contribue à faciliter le dialogue, le transfert de connaissances et donc la recherche de solutions qui pourront satisfaire lensemble des parties. Plusieurs acteurs issus de la société civile voyaient en les structures en place une façon de s'inviter au processus décisionnel; la déception venait souvent du fait que plusieurs avaient finalement l'impression que leur point de vue nétait pas intégré à la décision finale. 
Dans les régions qui nous ont intéressées, la question de la représentativité, importante pour assurer une participation publique de qualité, la légitimité de la procédure et son acceptabilité (voir Rowe and Frewer 2000), ne semble pas poser problème. En général, les principaux groupes d’acteurs sont présents, à l'exception peut-être des autochtones. La question de la participation autochtone dans la gestion des forêts au Québec est fort complexe et déborde le cadre de notre étude. Cette question est abordée plus spécifiquement par Fortier et Wyatt (2014) dans le cas des Atikamekw Nehirowisiwok. La présence de la plupart des acteurs au sein de ces lieux de concertation ne constitue cependant pas le seul critère pour juger de la participation. Pour que la participation publique soit efficace, il faut que les acteurs en présence développent une compréhension partagée des enjeux, un vocabulaire commun (Webler and Tuler 2001). Nous avons vu que selon les régions et les instances de concertation, ce nétait pas toujours le cas au Québec.

Le caractère exploratoire de cette étude était justifié en raison de la situation transitoire à laquelle nous nous attardions. Lanalyse proposée porte ainsi sur les réactions exprimées au moment du passage d'un cadre de concertation à un autre, et appelle ainsi à la poursuite détudes plus exhaustives alors que les nouvelles structures sont mieux définies. Nous tenons à rappeler que cette étude n'avait pas pour objectif d’offrir un portrait représentatif des perceptions de l'ensemble des intervenants dans les régions ciblées. Si certains groupes sont peu ou pas représentés dans l'étude (p. ex. autochtones, industriels forestiers), les avis, opinions et perceptions recueillis permettent néanmoins de porter un regard rétrospectif et prospectif sur les expériences de concertation en foresterie au Québec en contexte de changement du cadre institutionnel.

\section{Conclusion}

Si l'importance de la participation publique dans la gouvernance des ressources et du territoire forestier ne fait plus de doute au Canada, définir un modèle adéquat en fonction des réalités de chacune des régions demeure un défi. Dans le contexte québécois, l'arrimage des modes de participation au développement régional et à la gouvernance forestière semble avoir permis des avancées notables dans les régions éloignées des grands centres comme la Gaspésie et la Mauricie. Si ces avancées sont difficilement quantifiables, du moins dans le cadre de cette recherche, elles ressortent cependant clairement en ce qui a trait à la construction d'un dialogue entre les acteurs de la société civile, le Ministère et l'industrie forestière, ainsi quà l'augmentation des capacités des intervenants issus de la plupart des milieux concernés.

Lapproche adoptée par Québec, tant dans la mise en place des CRÉ et CRRNT que dans leur abolition décrétée de façon unilatérale, ne semble pas tenir compte des différences régionales. Alors que dans certaines régions, cette structure semblait s'ajouter à d'autres, déjà nombreuses, dans des régions comme la Gaspésie et la Mauricie, elle était considérée comme un forum incontournable pour les représentants sectoriels de lenvironnement et de la foresterie. Ainsi, il est essentiel de rappeler la tension historique entre la régionalisation par le haut, c'est-à-dire, le projet technocratique du gouvernement du Québec qui visait la mise en place de structures uniformes à l'ensemble des régions, et la régionalisation par le bas, soit un projet de prise en charge du développement par la société civile (Lévesque 2004). Si dans certaines régions, l’abolition des CRÉ simplifie les structures, dans d'autres, elle porte atteinte aux capacités de la société civile.

Cest à l'aulne des évènements récents que nous avons recueilli les perceptions sur létat actuel et anticipé de la participation publique en matière de gestion forestière dans quatre régions du Québec. Si de nombreux mécanismes demeurent en place pour assurer la participation des intervenants forestiers et de la société dans son ensemble, labolition des CRÉ restreint dans certaines régions les canaux de participation et de communication, et s'inscrit dans un contexte de diminution des ressources financières allouées au développement régional. La courte histoire de participation publique dans la foresterie au Québec semble être concluante à plusieurs égards. Toutefois, le gouvernement tarde, encore davantage avec l'abolition des CRÉ, à passer résolument de la consultation à une collaboration effective. Une collaboration, voir une coopération entre les acteurs d'une région donnée, permettrait de pérenniser les modalités du nouveau régime forestier. Alors que les structures de participation publique et de concertation sont redéfinies dans la plupart des régions du Québec, il importe de poursuivre les recherches en la matière afin de mieux cerner les spécificités régionales et les besoins qui en découlent.

\section{Remerciements}

Nous tenons à remercier Frédérik Doyon (Université du Québec en Outaouais), Guy Chiasson (Université du Québec en Outaouais) et Guy Lessard (Centre d'enseignement et de recherche en foresterie) pour leur aide dans la construction de létude et le recrutement de participants. Merci également à Luc Bouthillier pour ses commentaires constructifs suivant la lecture d'une version préliminaire de l'article. Nous reconnaissons la contribution financière du Centre d'enseignement et de recherche en foresterie et de Mitacs dans le cadre du programme Accélération.

\section{Bibliographie}

Agrawal, A., A. Chhatre and R. Hardin. 2008. Changing Governance of World's Forests. Science 320(13 June): 1460-1462.

Andersson, K. 2006. Understanding decentralized forest governance: An application of the institutional analysis and development framework. Sustainability: Science, Practice, \& Policy 2(1): 25-35.

Bachand, M. 2008. Les commissions forestières régionales et les commissions régionales sur les ressources naturelles et le territoire comme nouveau modèle de gouvernance de la ressource forestière au Québec, Mémoire de maîtrise, Centre universitaire de formation en environnement, Université de Sherbrooke, Sherbrooke.

Bäckstrand, K., J. Khan, A. Kronsell and E. Lövbrand. 2010. The promise of new modes of environmental governance. In : K. Bäckstrand, J. Khan, A. Kronsell et E. Lövbrand (eds.) Environmental Politics and Deliberative Democracy: Examining the Promise of New Modes of Governance. pp. 3-27. Edward Elgar Publishing Ltd, Cheltenham.

Beckley, T., J. Parkins and S. Sheppard. 2005. Public Participation in Sustainable Forest Management: A Reference Guide. Sustainable Forest Management Network, Edmonton, Alberta.

Belley, S. 2008. La construction politique des territoires. In : Guy Massicotte (ed.) Sciences du territoire. Perspectives québécoises. pp. 233-256. Presses de l'Université du Québec, Québec.

Blais, R. et G. Chiasson. 2005. L'écoumène forestier canadien: État, techniques et communautés - l'appropriation difficile du territoire. Revue canadienne des sciences régionales/Canadian Journal of Regional Science XXVIII(3): 487-512. 
Blais, R. et J. L. Boucher. 2013. Les temps des régimes forestiers au Québec. In : G. Chiasson et E. Leclerc (éds.) La gouvernance locale des forêts publiques au Québec. Une avenue de développement des régions périphériques? pp. 33-64. Presses de l'Université du Québec, Québec.

Bouthillier, L. et A. Roberge. 2007. Les intentions des programmes de participation du public appliqués par l'industrie forestière: État de la situation au Québec. The Forestry Chronicle 83(6): 810-817.

Bruna-Garcia, X. and M.F. Marey-Perez. 2014. Public participation: A need of forest planning. iForest 7: 216-226.

Buchy, M. and S. Hoverman. 2000. Understanding public participation in forest planning: A review. Forest Policy and Economics 1(1): 15-25.

Chiasson, G. et M. Robitaille. 2007. Les Conférences régionales des élus ou la démocratie revisitée. L'Observatoire en économie sociale, en développement régional et en organisation communautaire, Université du Québec en Outaouais. http://www4.uqo.ca/observer/DevLocal/Gouvernance/CRE.htm

Chiasson, G. et É. Leclerc. 2013. La gouvernance locale des forêts publiques au Québec. Une avenue de développement des régions périphériques? Presses de l'Université du Québec, Québec.

Chiasson, G., C. Andrew et J. Perron. 2006. Développement territorial et forêts: La création de nouveaux territoires forestiers en Abitibi et en Outaouais. Recherches sociographiques, 47(3): 555-572.

Chiasson, G., C. Gonzalez et É. Leclerc. 2011. La gouvernance participative des forêts publiques : L'Ontario et le Québec, des chemins parallèles ? Territoire en mouvement - Revue de géographie et aménagement [En ligne], 11 | 2011, mis en ligne le 01 septembre 2013 URL : http://tem.revues.org/1186

Chiasson, G., J.-P. Bernard et M. Charlebois. 2013. Le régime forestier. In : Y. Fournis, M.-J. Fortin, G. Brisson et G. Chiasson (éds.) La transition des régimes de ressources au prisme de la gouvernance: Les secteurs forestiers, porcins et éolien. Étude S1-3 remise au Comité d'évaluation environnementale stratégique des gaz de schiste et au MDDEFP.

Cloke, P., I. Cook, P. Crang, M. Goodwin, J. Painter and C. Philo. 2004. Practicing Human Geography. SAGE Publications Ltd, London, UK.

Coulombe, G., J. Huot, J.Arsenault, E. Bauce, J.-T. Bernard, A. Bouchard, M.-A. Liboiron et G. Szaraz. 2004. Commission détude sur la gestion de la forêt publique québécoise. Rapport final.

de Sardan, J.-P.O. 1995. La politique du terrain: Sur la production des données en anthropologie. Enquête 1 [En ligne] http:// enquete.revues.org/263.

Desrosiers, R., S. Lefebvre, P. Munoz et J. Pâquet. 2010. Guide sur la gestion intégrée des ressources et du territoire : son application dans lélaboration des plans d’aménagement forestier intégré. Ministère des Ressources naturelles et de la Faune, Québec.

Elo, S. and H. Kyngäs. 2007. The qualitative content analysis process. Journal of Advanced Nursing 62(1): 107-115.

Fortier, J.-F. et S. Wyatt. 2014. Cooptation et résistance dans la planification forestière concertée au Québec : Le cas des Atikamekw Nehirowisiwok et des "tables GIRT". Recherches amérindiennes au Québec 44(1): 35-47.

Fréchette, A. 2009. La gouvernance forestière au Québec : Le défi du changement institutionnel dans les systèmes socio-écologiques interdépendants. VertigO - la revue électronique en sciences de l'environnement Hors série(6), [En ligne] http://vertigo.revues. org/8948.

Hardy, R. et N. Séguin. 2011. Forêt et société en Mauricie. La formation d'une région. Septentrion, Québec.

Harvey, C. 2016. Programme d'aménagement durable des forêts Le ministre Laurent Lessard annonce un investissement de huit millions de dollars. 17 juillet 2015. URL: http://www.fil-information.gouv.qc.ca/Pages/Article.aspx?idArticle=2307176215 (accédé le 20 novembre 2016).
Hilsop, M., M. Twery and H. Vihemäki. 2004. Involving People in Forestry. A toolbox for Public Involvement in Forest and Woodland Planning. Forestry Commission, Bristol, UK.

Hoggart, K., L. Lees and A. Davies. 2002. Researching human geography. Arnold, London, UK.

Howlett, M. and J. Rayner. 2006. Globalization and Governance Capacity: Explaining Divergence in National Forest Programs as Instances of "Next-Generation" Regulation in Canada and Europe. Governance: An International Journal of Policy, Administration, and Institutions 19(2): 251-275.

Hsieh, H.-F. and S. E. Shannon. 2005. Three Approaches to Qualitative Content Analysis. Qual. Health Res. 15(9): 1277-1288.

ILO (2000). Public participation in forestry in Europe and North America: Report of the team of specialists on participation in forestry. Report WP 163, FAO/ECE/ILO Joint Committee Team, International Labour Office, Geneva, Switzerland.

Johansson, J. 2016. Participation and deliberation in Swedish forest governance: The process of initiating a National Forest Program. Forest Policy Econ. 70: 137-146.

Kangas, A., N. Saarinen, H. Saarikoski, L.A. Leskinen, T. Hujala and J. Tikkanen. 2010. Stakeholder perspectives about proper participation for Regional Forest Programmes in Finland. Forest Policy Econ. 12(3): 213-222.

Larson, A. M. and F. Soto. 2008. Decentralization of Natural Resource Governance Regimes. Annu. Rev. Env. Resour. 33: 213-239. Lawrence, R.L. and D. A. Deagen. 2001. Choosing public participation methods for natural resources: A context-specific guide. Soc. Natur. Resour. 14: 857-872.

Leclerc, É. et C. Andrew. 2013. Les tables de concertation de gestion intégrée des ressources forestières: est-ce que les GIR gouvernent? In : G. Chiasson et É. Leclerc (éds.) La gouvernance locale des forêts publiques au Québec. Une avenue de développement des régions périphériques? pp. 127-146. Presses de l'Université du Québec, Québec.

Lecomte, N., C. Martineau-Delisle and S. Nadeau. 2005. Participatory requirements in forest management planning in Eastern Canada: A temporal and interprovincial perspective. Forest. Chron. 81(3): 398-402.

Lévesque, B. 2004. Le modèle québécois et le développement régional et local: Vers un néolibéralisme et la fin du modèle québécois? Cahiers du CRISES Collection «Working Papers» no 0405.

MAMOT. 2016. (Ministère des Affaires municipales et de l'Occupation du territoire). Acteurs et processus - MRC. URL: http:// www.mamot.gouv.qc.ca/amenagement-du-territoire/guide-la-prisede-decision-en-urbanisme/acteurs-et-processus/mrcl (accédé le 8 décembre 2016).

Martineau-Delisle, C. 2013. La participation publique et la gestion des forêts au Québec: Changements de gouvernance, impacts des pratiques et profil des participants, thèse de doctorat, Département de sociologie, Université Laval, Québec.

Martineau-Delisle, C. and S. Nadeau. 2010. Assessing the effects of public participation processes from the point of view of participants: Significance, achievements, and challenges. Forest. Chron. 86(6): 753-765.

McGurk, B., A.J. Sinclair and A. Diduck. 2006. An Assessment of Stakeholder Advisory Committees in Forest Management: Case Studies from Manitoba, Canada. Soc. Natur. Resour. 19(9): 809-826. MFFP 2016. (Ministère des Forêts, de la Faune et des Parcs). Forêts [en ligne]. Disponible à https://mffp.gouv.qc.ca/les-forets/ [consultée le 7 novembre 2016].

MRN 2013. (Ministère des Ressources naturelles). Loi sur l'aménagement durable du territoire forestier - Une gouvernance renouvelée. Gouvernement du Québec, Québec. Disponible en ligne à: http://www.mffp.gouv.qc.ca/publications/forets/comprendre/ficheloi-amenagement-durable.pdf 
Pappila, M. 2013. Forest certification and trust-different roles in different environments. Forest Policy Econ. 31: 37-43.

Pappila, M. and I. Pölönen. 2012. Reconsidering the role of public participation in the Finnish forest planning system. Scand. J. Forest Res. 27(2): 177-185.

Parkins, J.R. and R.E. Mitchell. 2005. Public participation as public debate: A deliberative turn in natural resource management. Soc. Natur. Resour. 18(6): 529-540.

Parkins, J.R., S. Nadeau, L. Hunt, J. Sinclair, M. Reed et S. Wallace. 2006. Participation publique à la gestion forestière : Résultats d'un sondage national auprès de comités consultatifs. Ressources naturelles Canada, Service canadien des forêts, Centre de foresterie du Nord, Edmonton (Alberta). Rapport d'information NOR-X-409.

Primmer, E. and S. Kyllönen. 2006. Goals for public participation implied by sustainable development, and the preparatory process of the Finnish National Forest Programme. Forest Policy Econ. 8: 838-853

Reed, M.S. 2008. Stakeholder participation for environmental management: A literature review. Biol. Conserv. 141(10): 2417-2431.

Reed, M.S., A. Graves, N. Dandy, H. Posthumus, K. Hubacek, J. Morris, C. Prell, C.H. Quinn and L.C. Stringer. 2009. Who's in and why? A typology of stakeholder analysis methods for natural resource management. J. Environ. Manag. 90(5): 1933-1949.

Ribot, J.C., A. Agrawal and A.M. Larson. 2006. Recentralizing while decentralizing: How national governments reappropriate forest resources. World Devel. 34(11): 1864-1886.
Rowe, G. and L.J. Frewer. 2000. Public participation methods: A framework for evaluation. Sci. Technol. Human Values 25(1): 3-29. Saldaña, J. 2013. The Coding Manual for Qualitative Researchers. 2nd ed. Sage Publications Ltd, London, UK.

Smith, J.W., J.E. Leahy, D.H. Anderson and M.A. Davenport. 2013. Community/agency trust and public involvement in resource planning. Soc. Natur. Resour. 26(4): 452-471.

St-Hilaire, W.G.A. 2013. Réforme des politiques forestières au Québec et dynamique interne des structures étatiques de gestion forestière. In : G. Chiasson et É. Leclerc (éds.) La gouvernance locale des forêts publiques au Québec. Une avenue de développement des régions périphériques? pp. 65-88. Presses de l'Université du Québec, Québec.

Trudel, L., C. Simard et N. Vonarx. 2007. La recherche qualitative est-elle nécessairement exploratoire? Recherches qualitatives Hors série(5): 38-45.

Webler, T. and S. Tuler. 2001. Public participation in watershed management planning: Views on process from people in the field. Hum. Ecol. Rev. 8(2): 29-39.

Zhang, Y. and B. M. Wildemuth. 2009. Qualitative analysis of content. In: B.M. Wildemuth (ed.) Applications of Social Research Methods to Questions in Information and Library Science. pp. 308-319. Libraries Unlimited, Westport, CT.

Annexe 1 - Catégories, codes et sous-codes issus de l'analyse des entretiens

\begin{tabular}{|c|c|c|}
\hline Catégorie & Code & Sous-code \\
\hline \multirow[t]{3}{*}{$\begin{array}{l}\text { Participation publique et dynamique } \\
\text { de concertation régionale (avant } \\
\text { labolition des CRÉ) }\end{array}$} & Organisation des lieux de concertation & $\begin{array}{l}\text { Cadre réglementaire } \\
\text { Historique } \\
\text { Mission } \\
\text { Structure et fonctionnement }\end{array}$ \\
\hline & Participation des acteurs & $\begin{array}{l}\text { Ressources disponibles } \\
\text { Compétences } \\
\text { Communication } \\
\text { Outils de mobilisation } \\
\text { Relations informelles }\end{array}$ \\
\hline & Pertinence & $\begin{array}{l}\text { Pour l'organisme } \\
\text { Pour la région }\end{array}$ \\
\hline \multirow[t]{3}{*}{$\begin{array}{l}\text { Résilience des lieux de concertation } \\
\text { (après l'abolition des CRÉ) }\end{array}$} & Loi 28 & $\begin{array}{l}\text { Compréhension de la loi } \\
\text { Pertinence par rapport à la réalité régionale }\end{array}$ \\
\hline & Abolition des CRÉ & $\begin{array}{l}\text { Impacts sur la concertation } \\
\text { Impacts sur les activités de lorganisme }\end{array}$ \\
\hline & Avenir de la concertation & $\begin{array}{l}\text { Volonté de maintenir la concertation } \\
\text { Capacité régionale à maintenir la concertation } \\
\text { Rôle des MRC et des élus } \\
\text { Importance des relations informelles } \\
\text { Avenues envisagées }\end{array}$ \\
\hline
\end{tabular}

